Abanico Veterinario. Enero-Diciembre 2020; 10:1-17. http://dx.doi.org/10.21929/abavet2020.36 Artículo Original. Recibido: 23/03/2020. Aceptado: 25/11/2020. Publicado: 15/12/2020. Clave:2020-46.

\title{
Empleo de ácidos orgánicos en el agua de bebida y su efecto en el desempeño productivo en pollos de engorda
}

\author{
Organic acids employment in water and its effect on productive performance in broiler \\ chicks
}

\author{
Arce-Menocal José1 ID, Roa-Flores Martín ${ }^{1 \text { ID, }}$ López-Coello Carlos ${ }^{21 D}$, Ávila-

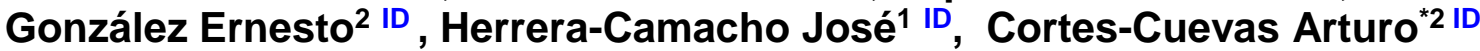

${ }^{1}$ Departamento de Avicultura, Facultad de Medicina Veterinaria y Zootecnia, Universidad Michoacana de San Nicolás de Hidalgo, Unidad Acueducto. Avenida Acueducto esq. con Tzintzuntzan, S/N, col. Matamoros, CP. 58240, Morelia Michoacán. Unidad Posta. Carretera Morelia-Zinapácuaro, km. 9.5, Municipio Tarímbaro Michoacán. ${ }^{2}$ Departamento de Medicina y Zootecnia de Aves, Facultad de Medicina Veterinaria y Zootecnia, Universidad Nacional Autónoma de México, Avenida Universidad No. 3000, Colonia UNAM, CU, Alcaldía Coyoacán, CP 04510 *Autor de correspondencia: Arturo Cortes Cuevas. Avenida Universidad No. 3000, Colonia UNAM, CU, Alcaldía Coyoacán, CP 04510 Autor Responsable: José Arce Menocal. Unidad Acueducto. Avenida Acueducto esq. con Tzintzuntzan, S/N, col. Matamoros, CP. 58240, Morelia Michoacán. Unidad Posta. Carretera Morelia-Zinapácuaro, km. 9.5, Municipio Tarímbaro Michoacán. josearce_55@yahoo.com, martinroa75@hotmail.com, coelloca@unam.mx, josheca@hotmail.com, avilaernesto@yahoo.com, cortescuevasarturo@yahoo.com.

\section{RESUMEN}

Se evaluó el empleo de ácidos orgánicos (AO) en el agua de bebida en pollo de 1 a 42 días de edad en el desempeño productivo, salud intestinal, $\mathrm{pH}$, morfología digestiva, $\mathrm{pH}$ sanguíneo, resistencia de yeyuno (RY) y pigmentación de piel (PP). Se utilizaron 1080 pollos distribuidos en tres tratamientos con nueve repeticiones de 40 aves: Los tratamientos fueron: Uso de agua de bebida sin acidificar $(\mathrm{pH}$ 8) grupo testigo (T1), acidificación con una mezcla AO (ácido fórmico 31\%, ácido propiónico 19\%, formato de amonio $26 \%$ y propionato de amonio 6\%) en dosis de $1.0 \mathrm{~L} / 1000 \mathrm{~L}$ de agua (T2) para obtener un $\mathrm{pH}$ de 4 y $0.3 \mathrm{~L} / 1000 \mathrm{~L}$ de agua (T3) para un $\mathrm{pH}$ de 6 . Los resultados mostraron $(\mathrm{p} \leq 0.01)$ mayor peso corporal, menor consumo de alimento y mejor eficiencia alimenticia, para las aves del T3. No existieron diferencias $(p \geq 0.05)$ en los valores del pH digestivo, PP y morfología intestinal; únicamente mostraron efectos $(p \leq 0.01)$ en el ancho de vellosidades y en el área digestiva. El pH sanguíneo y la $R Y$, mostraron efectos $(p \leq 0.01)$ entre los tratamientos. Se concluye que la acidificación en el agua de bebida en pollos de engorda, con la mezcla $\mathrm{AO}$ en $0.3 \mathrm{~L} / 1000 \mathrm{~L}$ es suficiente para lograr un mejor desempeño productivo.

Palabras clave: Pollo de engorda, ácidos orgánicos, rendimiento productivo.

\begin{abstract}
In order to evaluate the productive performance, gut health, $\mathrm{pH}$, digestive morphology, blood $\mathrm{pH}$, jejunum resistance (RY) and skin pigmentation (PP) on the employment of an additional mixture of organic acids (AO) into the water, in broilers from 1 to 42 days of age, 1080 chickens were used, distributed in three treatments with nine repetitions of 40 birds. The treatments used: non-acidified drinking water $(\mathrm{pH} 8)$ control group (T1), acidification with an AO mixture (formic acid $31 \%$, propionic acid $19 \%$, ammonium format $26 \%$ and ammonium propionate $6 \%$ ) in a dose of $1.0 \mathrm{~L} \mathrm{/} 1000 \mathrm{~L}$ of water (T2) to obtain a pH of 4 , and $0.3 \mathrm{~L} /$ $1000 \mathrm{~L}$ of water (T3) for a pH of 6 . The results showed ( $p \leq 0.01$ ) higher body weight, lower feed intake, and better feed efficiency for the birds from T3. There were no differences $(p \geq 0.05)$ on the values of digestive $\mathrm{pH}, \mathrm{PP}$, and intestinal morphology; only showed effects $(\mathrm{p} \leq 0.01)$ in the width of villi and in the digestive area. Blood $\mathrm{pH}$ and $\mathrm{RY}$ showed effects $(\mathrm{p} \leq 0.01)$ among treatments. It is concluded that the acidification of the drinking water in broiler chickens, with the $A O$ mixture at $0.3 \mathrm{~L} / 1000 \mathrm{~L}$ is sufficient to obtain better performance.
\end{abstract}

Keywords: Broilers, organic acids, performance. 


\section{INTRODUCCIÓN}

Los compuestos conocidos como ácidos orgánicos (AO), utilizados en la industria pecuaria, son ácidos carboxílicos alifáticos formados por carbono, oxígeno e hidrógeno, también son llamados ácidos grasos de cadena corta (AGCC) o ácidos grasos volátiles; cuya cadena estructural está compuesta por menos de 7 moléculas de carbono; estos compuestos son utilizados como una de las alternativas al empleo de antibióticos promotores del crecimiento en la industria avícola (Ricke, 2003; Khan e lqbal, 2016). Comercialmente los $\mathrm{AO}$ como el ácido propiónico, han sido utilizados por más de 30 años para reducir el crecimiento bacteriano y de hongos en el alimento, para preservar de manera higiénica la calidad del mismo; así como prevenir y controlar infecciones por Salmonella spp y E. coli K88 en las aves y sus derivados (Freitag, 2007; Emami et al., 2017). Por otro lado, a causa de la prohibición en el uso de antibióticos en los alimentos de animales por regulaciones en la comunidad Europa y Estados Unidos (Smith, 2011), los $A O$ han demostrado ser una alternativa en la modulación de la microbiota entérica e inhibición de bacterias patógenas intestinales, como E coli, Salmonella Typhimurium y Campylobacter coli; además de promover la colonización de flora benéfica como los lactobacilos (Roth et al., 2017; Roth et al., 2019; Bourassa et al., 2018; Mortada et al., 2020).

Por otro lado, algunos estudios muestran el impacto positivo en los parámetros productivos, integridad intestinal, respuesta inmune y microbiota intestinal (Emami et al., 2017; Polycarpo et al., 2017; Araujo et al., 2018; Nguyen et al., 2018; Adhikari et al., 2020; Al-Mutairi et al., 2020), beneficios que inicialmente han sido atribuidos al efecto que tienen sobre el ambiente del tracto gastrointestinal (TGl), en el proceso de digestión y como fuente de energía; pero principalmente como protección del mismo. Los AO se encuentran de manera natural en TGI de las aves; entre los que se incluyen ácido láctico en mayor proporción en el intestino delgado; mientras que los ácidos propiónico, acético y butírico se localizan mayormente en los sacos ciegos; esto es debido al proceso de fermentación (Meimandiopur et al., 2011; Khan e lqbal, 2016; Rhot et al., 2017). Las propiedades químicas, los efectos en la reducción del $\mathrm{pH}$ y la eficacia de la inhibición microbiana de un ácido dependen de su valor pKa, que es el pH al cual los $\mathrm{AO}$ están disociados al $50 \%$. El valor del pKa define el poder de acción que pueden tener los $A O$; mientras más bajo es su valor, es considerado con una mayor capacidad de acidificar (Freitag, 2007; Khan e lqbal, 2016).

La teoría más aceptada sobre el mecanismo de acción en la inhibición del crecimiento de bacterias por parte de los $\mathrm{AO}$, considera su liposolubilidad en medios con $\mathrm{pH}$ ácido; un $\mathrm{pH}$ cercano a 4.5 mantiene la liposolubilidad del compuesto, mismo que les permite penetrar a la célula bacteriana y disociarse en su citoplasma que generan un desbalance metabólico que concluye con la muerte del microorganismo (Nakal y Siebert, 2003; Immerseel et al., 2006) El ácido propiónico combinado con el ácido fórmico al ser 
utilizados en el alimento, han demostrado sinergia en el control de Salmonella spp, Campylobacter spp y E. coli (Roth et al., 2017; Emami et al., 2017; Adhikari et al., 2020); así como beneficios zootécnicos. La inclusión de ácido fórmico en combinación con ácido propiónico vía agua de bebida (Nhuyen et al., 2018; Adhikari et al., 2020), mostraron resultados favorables en los parámetros en el pollo de engorda de 1 a 35 días de edad, donde incluyeron $0.02,0.03,0.04,0.05$ y $0.06 \%$ de ácidos orgánicos.

Sin embargo, la información no menciona los valores de $\mathrm{pH}$. Otras investigaciones en pollos utilizaron una mezcla de ácidos orgánicos (ácido propiónico, ácido fórmico y ácido butírico), para acidificar el agua a un pH de 3 a 4.5; los resultados arrojaron beneficios en los parámetros productivos, microbiota intestinal, respuesta inmune, digestibilidad de nutrientes y calidad de la canal (Ghulam et al., 2013; Emami et al., 2017; Polycarpo et al., 2017; Araujo et al., 2018; Nguyen et al., 2018; Adhikari et al., 2020; Al-Mutairi et al., 2020 ); sin embargo, la literatura disponible sobre el uso de estos $A O$ utilizados en el agua de bebida y dirigidos a demostrar sus beneficios sobre el desempeño zootécnico y $\mathrm{pH}$ intestinales, son escasos.

El objetivo del presente trabajo fue evaluar el desempeño zootécnico del pollo de engorda, a la adición de una mezcla de $\mathrm{AO}$ en el agua de bebida durante su vida productiva; así como el pH digestivo y morfología del duodeno, pH sanguíneo, resistencia a la tracción del yeyuno y amarillamiento de la piel.

\section{Localización}

\section{MATERIAL Y MÉTODOS}

El trabajo se realizó en una granja avícola experimental localizada en el municipio de Charo, estado de Michoacán, a una altura de 1,940 metros sobre el nivel del mar, con una temperatura mínima anual de $16^{\circ} \mathrm{C}$ y una máxima de $18^{\circ} \mathrm{C}$; la precipitación pluvial máxima es de 800 mm y mínima de 600 mm (Municipio de Charo, 2020).

\section{Animales}

Todos los procedimientos utilizados en el manejo de los animales fueron aprobados por el comité del cuidado y bienestar animal de la Facultad de Medicina Veterinaria y Zootecnia de la UNAM.

\section{Diseño experimental}

Se utilizaron 1080 pollitos de ambos sexos (50\% machos y 50\% hembras), de 1 día de edad de la estirpe Ross 308 de una incubadora comercial (El Avión), ubicada en Tepic, Nayarit., los cuales se mantuvieron hasta los 42 días de edad en los meses de abril y mayo. Fueron distribuidos aleatoriamente en tres tratamientos, con nueve réplicas de 40 aves. Los tratamientos consistieron en la administración de una mezcla de $\mathrm{AO}$, al agua de bebida para llegar a diferentes $\mathrm{pH}$, desde la llegada del pollito hasta el final de la prueba en forma continua. Los $\mathrm{AO}$ administrados fueron una mezcla comercial fabricado por Novus International (Acidomix ${ }^{\circledR}$ AFL, Querétaro, México), compuesta de una combinación de ácido fórmico 31\%, ácido propiónico 19\%, formato de amonio $26 \%$ y 
propionato de amonio 6\%. Previo al experimento, se analizó la calidad del agua de la granja por parte de la "Comisión Nacional de Agua" (localizada en Morelia, Michoacán, México), para conocer, las condiciones fisicoquímicas de la fuente y corroborar su viabilidad para su uso en animales domésticos (cuadro 1). El pH del agua fue medido con un potenciómetro portátil Hanna HI-98127.

Cuadro 1. Análisis Fisicoquímico del Agua*

\begin{tabular}{|c|c|c|c|c|}
\hline Parámetros & Unidades & $\begin{array}{c}\text { Niveles } \\
\text { admisibles } \\
\text { en aves }\end{array}$ & $\begin{array}{c}\text { Toma } \\
\text { municipal }\end{array}$ & $\begin{array}{c}\text { Grado de } \\
\text { cumplimiento }\end{array}$ \\
\hline Temperatura & ${ }^{\circ} \mathrm{C}$ & & 25 & \\
\hline Potencial hidrógeno & $\mathrm{pH}$ & $6.5-8.5$ & 8.0 & Cumple \\
\hline Conductividad eléctrica & $\mu \mathrm{ohms} / \mathrm{cm}$ & & 526 & \\
\hline Turbiedad & UTN & & 1.4 & \\
\hline Color & Pt-Co & & 5 & \\
\hline Oxígeno disuelto & $\mathrm{mg} / \mathrm{L}$ & & 6.4 & \\
\hline Demanda bioquímica de oxígeno & $\mathrm{mg} / \mathrm{L}$ & & 2.4 & \\
\hline Demanda química de oxígeno & $\mathrm{mg} / \mathrm{L}$ & & 5 & \\
\hline Sólidos sedimentables & $\mathrm{mg} / \mathrm{L}$ & & 0 & \\
\hline Sólidos totales & $\mathrm{mg} / \mathrm{L}$ & & 415 & \\
\hline Sólidos suspendidos totales & $\mathrm{mg} / \mathrm{L}$ & & 20 & \\
\hline Sólidos disueltos totales & $\mathrm{mg} / \mathrm{L}$ & 1000 & 395 & Cumple \\
\hline Nitratos & $\mathrm{mg} / \mathrm{L}$ & 10 & 0.2 & Cumple \\
\hline Nitrogeno amoniacal & $\mathrm{mg} / \mathrm{L}$ & $60-80$ & 0.5 & \\
\hline Dureza total & $\mathrm{mg} / \mathrm{L}$ & & 118.9 & Cumple \\
\hline Dureza de calcio & $\mathrm{mg} / \mathrm{L}$ & & 82 & \\
\hline Dureza de magnesio & $\mathrm{mg} / \mathrm{L}$ & & 36.9 & \\
\hline Alcalinidad total & $\mathrm{mg} / \mathrm{L}$ & & 200 & \\
\hline Alcalinidad a la fenolftaleina & $\mathrm{mg} / \mathrm{L}$ & & 0 & \\
\hline Cloruros & $\mathrm{mg} / \mathrm{L}$ & 200 & 46.7 & Cumple \\
\hline Sulfatos & $\mathrm{mg} / \mathrm{L}$ & 125 & 24.2 & Cumple \\
\hline Carbonatos & $\mathrm{mg} / \mathrm{L}$ & & 0 & \\
\hline Bicarbonatos & $\mathrm{mg} / \mathrm{L}$ & 60 & 200 & \\
\hline Calcio & $\mathrm{mg} / \mathrm{L}$ & & 32.8 & Cumple \\
\hline Magnesio & $\mathrm{mg} / \mathrm{L}$ & 32 & 8.9 & \\
\hline Sodio & $\mathrm{mg} / \mathrm{L}$ & 0 & 66.3 & Cumple \\
\hline Coliformes fecales & UFC/100ml & & 0 & Cumple \\
\hline
\end{tabular}

${ }^{*}$ Comision Nacional del Agua 
Los tratamientos se describen, como:

1) Un control sin $\mathrm{AO}$ ni promotor del crecimiento; se utilizó la red de agua potable del municipio de Charo, Michoacán para su consumo, el cual tenía un pH de 8.0 sin la adición de mezcla de $A O$.

2) Agua de bebida del tratamiento contro más la adición de la mezcla de $A O$ a razón de 1.0 litros por cada 1000 litros de agua, para establecer 0.128 moles y obtener un pH de 4.0

3) Agua de bebida del tratamiento control más la adición de la mezcla de AO a razón de 0.3 litros por cada 1000 litros de agua, para establecer 0.0038 Moles y obtener un $\mathrm{pH}$ de 6.0

Para calcular la concentración molar de la combinación de los ácidos orgánicos utilizados (cuadro 2), se identificó el peso molecular de cada uno de ellos, y se expresaron en milimoles (mM) descritos por Brown, 2002. Se obtuvo los gramos por kilo de la solución utilizada y se dividió entre el peso molecular para obtener los moles por kilo de la solución, el cual se dividió entre 1.1, que es la densidad específica de la mezcla de los ácidos utilizados, (considerar que un litro pesa más que un kilo). Se obtuvo la molaridad en gramos por litro de la mezcla de ácidos, lo que ayudó a calcular la dosis usada para bajar el pH a 4.0 y a 6.0 del agua con pH de 8.0. El cálculo se estableció mediante la adición a mil litros de agua del tratamiento control ( $\mathrm{pH}$ de 8.0); cantidades crecientes hasta lograr el $\mathrm{pH}$ de 4.0 y de 6.0. Se encontró la dosis de 1.0 litros de la mezcla de los ácidos orgánicos utilizados para $\mathrm{pH} 4.0$ y de 0.3 litro para $\mathrm{pH}$ de 6.0. Para conocer la cantidad de Moles utilizados, se obtuvo de la suma total de la mezcla de los ácidos de la molaridad multiplicada por la dosis usada dividida entre 1000. Así se obtuvo que para pH de 4.0 el valor fue de 0.128 y 0.0038 Moles para $\mathrm{pH}$ de 6.0 .

Se utilizó una caseta de 11 m de ancho x 40 m de largo, con techo de lámina galvanizada; con una capacidad de 27 pisos cada uno de ellos, acondicionados con cama de viruta de madera; dos comederos de tolva de $45 \mathrm{~cm}$ de diámetro en su base con capacidad para 10 kilos cada uno; así como un bebedero tipo Plasson automático redondo, el cual estaba conectado a un garrafón con capacidad de 20 litros graduado; en el cual se adicionaba la cantidad descrita de $\mathrm{AO}$ para llegar a mantener el $\mathrm{pH}$ requerido diariamente y que servía además para evaluar el consumo de agua por ave. Las dietas fueron formuladas en forma similar para cada uno de los tratamientos con una presentación en harina; se utilizó como base maíz-soya, en tres etapas (0-21; 22-35 y 36-42 días de edad), donde se cubrió las necesidades establecidas para la estirpe del pollo de engorda, (Ávila, 2018), proporcionado a libre acceso (cuadro 3), sin la adición de antibióticos como promotores de crecimiento (APC). 
Cuadro 2. Cálculo de la concentración molar de la combinación de los ácidos orgánicos utilizados para obtener el pH deseado en el agua

\begin{tabular}{|c|c|c|c|c|c|c|}
\hline Compuesto & $\begin{array}{c}\text { Peso } \\
\text { Molecular } \\
\text { en } \\
\text { Milimoles* }\end{array}$ & $\begin{array}{c}\text { g por Kg } \\
\text { de } \\
\text { solución }\end{array}$ & $\begin{array}{l}\text { moles } x \\
\text { Kg de } \\
\text { solución }\end{array}$ & $\begin{array}{l}\text { Molaridad g } \\
\text { x It de } \\
\text { solución }\end{array}$ & $\begin{array}{c}\mathrm{pH} 6 \\
\\
\left(0.3 \text { It } \mathrm{AFL}^{*} /\right. \\
1000 \text { It de } \\
\text { agua) }\end{array}$ & $\begin{array}{c}\mathrm{pH} 4 \\
\left(1.0 \text { It } \mathrm{AFL}^{*} /\right. \\
1000 \text { It de } \\
\text { agua) }\end{array}$ \\
\hline $\begin{array}{c}\text { Ácido } \\
\text { Propiónico }\end{array}$ & 74 & 190 & 2.567 & 2.336 & & \\
\hline $\begin{array}{l}\text { Ácido } \\
\text { Fórmico }\end{array}$ & 46 & 310 & 6.739 & 6.127 & & \\
\hline $\begin{array}{c}\text { Formato de } \\
\text { Amonio }\end{array}$ & 63 & 260 & 4.126 & 3.754 & & \\
\hline $\begin{array}{l}\text { Propionato } \\
\text { de Amonio }\end{array}$ & 91 & 60 & 0.659 & 0.600 & & \\
\hline Total AO & & 820 & 14.091 & 12.817 & 0.0038 & 0.0128 \\
\hline
\end{tabular}

Descritos por Brown (1) AFL. Producto utilizado

El programa de sanitario fue similar para todos los tratamientos; al primer día de edad en la planta incubadora se aplicó la vacuna contra Marek, y en la granja experimental se aplicaron dos vacunas contra la enfermedad de Newcastle, vía ocular cepa la Sota (al día 8 y 25 días de edad). Durante las primeras cuatro semanas las aves fueron criadas en una caseta equipada con criadoras automáticas infrarrojas de gas, y recibieron un programa de luz natural. El alimento y el agua de bebida se proporcionaron a libre acceso. A los 42 días de edad se resumieron los datos de registros de las aves mixtas (machos y hembras) de peso corporal (g), consumo de alimento acumulado (g), eficiencia alimenticia ( $\mathrm{g} / \mathrm{g}$ ), consumo de agua (litros) y mortalidad (\%). Se seleccionaron 18 machos de manera aleatoria por tratamiento ( 2 por réplica), para evaluar en aves vivas la pigmentación amarilla de la piel (en la vena de la grasa), con el fotocolorímetro de reflactancia CR-400 (Kónica Minolta Sensing, NJ, USA); bajo la escala CIELab del Comité Internacional de Colorimetría.

De cada ave se obtuvo una muestra de sangre de la vena yugular, para evaluar el pH sanguíneo. Posteriormente la matanza de las aves se realizó de acuerdo a lo establecido en la Norma Oficial Mexicana, por separación de la cabeza del cuerpo, con un objeto cortante; a través de un solo movimiento firme y certero NOM-033-ZOO-1995, NORMA Oficial Mexicana Sacrificio Humanitario de los Animales domésticos y silvestres (NOM, 1995). Para evaluar el pH in situ del tracto gastrointestinal, el cual se realizó de manera inmediata posterior al sacrificio, con un potenciómetro marca Fisher Scientific modelo $\mathrm{AB} 15 / 15+$. 
Cuadro 3. Análisis calculado de las dietas

\begin{tabular}{cccc}
\hline Nutrientes & 1-21 días & 22-35 días & 36-42 días \\
\hline Proteína Cruda (\%) & 22.00 & 20.1 & 18.5 \\
EM. Kcal./Kg. & 3025 & 3185 & 3210 \\
Lisina (\%) & 1.38 & 1.17 & 1.05 \\
Metionina (\%) & 0.64 & 0.59 & 0.52 \\
Metionina+Cistina (\%) & 1.00 & 0.94 & 0.83 \\
Treonina (\%) & 0.84 & 0.78 & 0.68 \\
Triptófano (\%) & 0.27 & 0.25 & 0.23 \\
Calcio (\%) & 1.0 & 0.94 & 0.85 \\
Fósforo Disponible (\%) & 0.46 & 0.40 & 0.38 \\
Sodio (\%) & 0.20 & 0.18 & 0.17 \\
\hline
\end{tabular}

La evaluación de la morfología histológica del duodeno, se realizó con cortes transversales de $5 \mathrm{~cm}$ del asa duodenal, los cuales se fijaron en formol al $10 \%$ para su procesamiento histológico y teñido por la técnica de hematoxilina y eosina. Una vez preparada la laminilla se midió en micras ( $\mu$ ), la longitud y el ancho de 5 vellosidades de cada muestra; además de la cripta de Lieberkuhn adyacente con apoyo del programa Motic Images Plus 2.0 (Routine Software Series, Motic Asia, Hong Kong). La fórmula estimada del área en duodeno fue, largo $x$ ancho de la vellosidad a nivel medio de la misma $\left(\mu^{2}\right)$, dividida entre 1000. Asimismo, fue evaluada la resistencia al rompimiento por tracción del yeyuno, con la ayuda de un dinamómetro digital (IMADA MV 110), en cortes de $10 \mathrm{~cm}$ previos al divertículo de Meckel; valores expresados en kilogramos-fuerza y transformados a la unidad internacional pascal por metro cuadrado.

Las medias resultantes de los parámetros productivos y mortalidad, así como las demás variables, se analizaron bajo un diseño de análisis de varianza; se utilizó el modelo lineal general y cuando existieron diferencias significativas ( $p \leq 0.05$ ), entre los tratamientos, se realizó la comparación de medias por la prueba de Tukey (SAS Institute Inc, 2012). Los resultados expresados en porcentajes fueron transformados a la proporción arco seno, para su análisis. 


\section{RESULTADOS}

Los resultados de los parámetros zootécnicos se muestran en el cuadro 4 . Se encontró efecto $(p \leq 0.05)$ mayor peso corporal $(2.7 \%)$, en el T2 con respecto al T1 control. Por otra parte, se disminuyó el consumo de alimento ( $p \leq 0.01$ ) en el tratamiento 3 , en $3.1 \%$ respecto al T1 Control y $2.7 \%$, con relación al T2; lo que mejoró significativamente ( $\mathrm{p} \leq$ 0.01 ), en los valores de eficiencia alimenticia con relación al T1 en $5.9 \%$ y en $3.8 \%$ al $\mathrm{T} 2$ con $\mathrm{pH}$ de 4. El consumo de agua fue menor $(\mathrm{p} \leq 0.01)$ en el grupo control (T1), donde no se adicionaron los $A O$ en el agua de bebida con relación a las aves que estuvieron consumiendo agua con AO (T2 y T3); lo que representó un incremento de 2.23 y $2.42 \%$ respectivamente. No se presentaron efectos $(p \geq 0.05)$ en el porcentaje de la mortalidad entre los tratamientos evaluados.

Los valores del $\mathrm{pH}$ gastrointestinal no presentaron efectos significativos ( $p \geq 0.05$ ), entre los tratamientos estudiados en los diferentes segmentos evaluados; como se muestra en el cuadro 5, se ve en general valores a nivel del promedio y/o por abajo, aquellas aves que consumieron agua con la adición de AO (T2 y T3).

Cuadro 4. Promedios y error estándar de los parámetros zootécnicos y mortalidad en el pollo de engorda con $\mathrm{AO}$, en el agua de bebida a los 42 días de edad

\begin{tabular}{|c|c|c|c|c|c|}
\hline $\begin{array}{c}\text { AO } \\
\text { (L/1000L) }\end{array}$ & $\begin{array}{c}\text { Peso corporal } \\
\text { (kg) }\end{array}$ & $\begin{array}{l}\text { Consumo de } \\
\text { alimento (kg) }\end{array}$ & $\begin{array}{l}\text { Eficiencia } \\
\text { alimenticia }\end{array}$ & $\begin{array}{c}\text { Consumo de } \\
\text { agua (L) }\end{array}$ & $\begin{array}{c}\text { Mortalidad } \\
(\%)\end{array}$ \\
\hline T1.- 0 & $2.46 \pm 0.011^{b}$ & $4.76 \pm 0.022^{a}$ & $0.51 \pm 0.01^{b}$ & $7.40 \pm 0.015^{a}$ & $6.3 \pm 0.8^{a}$ \\
\hline T2.- 0.3 & $2.53 \pm 0.007^{a}$ & $4.62 \pm 0.016^{b}$ & $0.54 \pm 0.0^{\mathrm{a}}$ & $7.57 \pm 0.029^{b}$ & $5.4 \pm 1.5^{\mathrm{a}}$ \\
\hline T3.- 1.0 & $2.49 \pm 0.016^{\mathrm{ab}}$ & $4.75 \pm 0.019^{a}$ & $0.52 \pm 0.0^{\mathrm{b}}$ & $7.58 \pm 0.028^{b}$ & $5.8 \pm 1.8^{a}$ \\
\hline PROMEDIO & $2.50 \pm 0.009$ & $4.71 \pm 0.018$ & $0.52 \pm 0.01$ & $7.51 \pm 0.024$ & $5.8 \pm 0.8$ \\
\hline Probabilidad & 0.006 & 0.001 & 0.001 & 0.001 & 0.919 \\
\hline
\end{tabular}

a, b. Literales distintas entre las columnas muestran diferencias significativas ( $p \leq 0.01)$

Las mediciones histológicas del duodeno (cuadro 6), no mostraron diferencias ( $p \geq 0.05$ ) en el largo de vellosidades y en la profundidad de criptas de Lieberkuhn, entre los tratamientos evaluados; en el ancho de las vellosidades se observaron diferencias ( $p \leq$ 0.01 ), con los valores más altos en el tratamiento 3 , con diferencias de 23 y 42 micras, con relación al T1 y T2; este efecto influyó en los resultados del área digestiva calculada, con los valores más altos ( $p \leq 0.01$ ), la morfología del tratamiento Control con pH de 8 y de 4. 
Cuadro 5. Valores del pH gastrointestinal en el pollo de engorda con el uso de AO, en el agua de bebida a los 42 días de edad

\begin{tabular}{|c|c|c|c|c|c|c|c|}
\hline $\mathrm{AO}$ (L/1000L) & Buche & Proventrículo & Molleja & Duodeno & Yeyuno & Íleon & Ciegos \\
\hline T1.- 0 & $5.3 \pm 0.1$ & $3.1 \pm 0.2$ & $3.2 \pm 0.2$ & $5.8 \pm 0.0$ & $6.0 \pm 0.1$ & $7.0 \pm 0.1$ & $6.3 \pm 0.2$ \\
\hline T2.- 0.3 & $4.8 \pm 0.2$ & $3.1 \pm 0.3$ & $3.1 \pm 0.2$ & $5.6 \pm 0.1$ & $5.8 \pm 0.1$ & $6.7 \pm 0.1$ & $6.0 \pm 0.1$ \\
\hline T3.- 1.0 & $5.0 \pm 0.3$ & $2.9 \pm 0.3$ & $2.6 \pm 0.3$ & $5.7 \pm 0.0$ & $5.7 \pm 0.2$ & $6.8 \pm 0.2$ & $6.0 \pm 0.1$ \\
\hline PROMEDIO & $5.0 \pm 0.1$ & $3.1 \pm 0.2$ & $3.0 \pm 0.1$ & $5.7 \pm 0.0$ & $5.8 \pm 0.1$ & $6.8 \pm 0.1$ & $6.1 \pm 0.1$ \\
\hline Probabilidad & 0.189 & 0.857 & 0.219 & 0.099 & 0.347 & 0.325 & 0.331 \\
\hline
\end{tabular}

Cuadro 6. Mediciones histológicas de duodeno en el pollo de engorda con el uso de AO, en el agua de bebida a los 42 días de edad

\begin{tabular}{|c|c|c|c|c|}
\hline Tratamientos & Largo $(\mu)$ & Ancho $(\mu)$ & Criptas $(\mu)$ & Área $\left(\mu^{2}\right) / 1000$ \\
\hline \multirow[t]{2}{*}{$A O(L / 1000 L)$} & \multicolumn{4}{|c|}{ Promedio \pm Error estándar } \\
\hline & $1500 \pm 12$ & $121 \pm 5^{b}$ & $169 \pm 8$ & $182 \pm 8.0^{\mathrm{a}}$ \\
\hline T1.- 0 & $1493 \pm 36$ & $102 \pm 5^{c}$ & $150 \pm 4$ & $153 \pm 8.2^{b}$ \\
\hline T2.- 0.3 & $1422 \pm 41$ & $144 \pm 7^{a}$ & $153 \pm 9$ & $203 \pm 8.9^{a}$ \\
\hline T3.- 1.0 & $1476 \pm 18$ & $121 \pm 4$ & $158 \pm 4$ & $177 \pm 5.4$ \\
\hline Probabilidad & 0.182 & 0.001 & 0.134 & 0.001 \\
\hline \multicolumn{5}{|c|}{$\begin{array}{l}{ }^{*} \text { a,b,c. Literales distintas marcan diferencias significativas }(\mathrm{p} \leq 0.01) \\
\text { El pH sanguíneo fue mayor ( } \mathrm{p} \leq 0.02) \text { en el } \mathrm{T} 1 \text {, en relación a los tratamientos } 3 \text { y } 2 \\
\text { respectivamente, los cuales manifestaron menores } \mathrm{pH} \text { sanguíneos; asimismo, la } \\
\text { resistencia a la tracción del yeyuno mostraron efectos }(\mathrm{p} \leq 0.03) \text {, entre los tratamientos } \\
\text { evaluados; siendo el tratamiento } 1 \text { el que mostró la resistencia más baja que los } \\
\text { tratamiento } 3 \text { y } 2 \text { respectivamente, sin mostrar efectos ( } \mathrm{P} \leq 0.05) \text { entre los tratamientos } \\
\text { evaluados en los valores de pigmentación amarilla en piel, como se observan en el cuadro } \\
7 \text {. }\end{array}$} \\
\hline
\end{tabular}


Cuadro 7. Promedios del pH sanguíneo, resistencia a la tracción del Yeyuno y pigmentación amarilla de la piel en el pollo de engorda con el uso de AO, en 42 días de edad

\begin{tabular}{cccc} 
& Valores Sanguíneos & Resistencia del yeyuno & Amarillamiento \\
Tratamientos & $\mathrm{pH}$ & Pascal $/ \mathrm{m}^{2}$ & Deltas \\
\hline
\end{tabular}

$\mathrm{AO}(\mathrm{L} / \mathbf{1 0 0 0 L})$

Promedio \pm error estándar

$\begin{array}{cccc} & 7.29 \pm 0.05^{\mathrm{b}} & 2.60 \pm 0.017^{\mathrm{b}} & 19.17 \pm 1.76 \\ \text { T1.- } 0 & 7.06 \pm 0.02^{\mathrm{a}} & 3.37 \pm 0.019^{\mathrm{a}} & 18.04 \pm 1.99 \\ \text { T2.- } \mathbf{0 . 3} & 7.16 \pm 0.03^{\mathrm{ab}} & 3.30 \pm 0.023^{\mathrm{ab}} & 19.45 \pm 0.72 \\ \text { T3.- } \mathbf{1 . 0} & \mathbf{7 . 1 6} \pm \mathbf{0 . 0 3} & \mathbf{3 . 1 1} \pm \mathbf{0 . 0 1 4} & \mathbf{1 8 . 8 4} \pm \mathbf{0 . 9 0 9} \\ \text { Probabilidad } & 0.002 & 0.033 & 0.812\end{array}$

$a, b=$ Literales distintas entre las columnas muestran diferencias significativas ( $\leq \leq 0.01)$

\section{DISCUSIÓN}

Los beneficios zootécnicos que se obtienen con la adición de $\mathrm{AO}$ se ha comprobado con diferentes compuestos, como los ácidos fumárico, fórmico, acético y propiónico, (Ghulam et al., 2013; Broom, 2015; Emami et al., 2017; Al-Mutairi et al., 2020); así como en la mezcla de éstos; donde la combinación del ácido fórmico con ácido acético y propionatos han demostrado tener un efecto positivo en el rendimiento productivo, morfología intestinal, respuesta inmune y en la microbiota intestinal (Adil et al., 2011; Polycarpo et al., 2017; Araujo et al., 2018; Nguyen et al., 2018). Históricamente, estos beneficios se han asociado de manera inicial a la modulación de la microflora nativa y patógena del tracto intestinal, demostrado por el efecto que tienen los $A O$ en agua de bebida $y$ alimento, para contrarrestar el efecto negativo de bacterias patógenas, como Salmonella spp, E. coli, Campylobacter spp y Clostridium spp., (Immersel et al., 2006; Emami et al., 2017; Adhikari et al., 2020; Bourassa et al., 2018; Mortada et al., 2020), mediante la colonización del tracto digestivo por Lactobacillus. Se consideran que éstos son resistentes a pH ácidos, teniendo un papel de protección en el tracto gastro-intestinal (Jin et al., 1998). Broom (2015) menciona que en un medio ácido de pH 4.6 el efecto de los AO como antibacterianos es más efectivo, con base a evaluaciones realizadas in vitro, en donde concluyen que la concentración mínima inhibitoria para los AO puede variar con el tipo de bacteria y ácido utilizado. Otros estudios in vitro con cultivos de Escherichia coli, (Dibner y Buttin, 2002), añaden diferentes ácidos, entre los que se incluyeron clorhídrico, fórmico, láctico e hidroxi-análogo de metionina (HMTBa), para acidificar el medio de cultivo a pH 4 y pH 7, demostró que el efecto antibacteriano es más eficiente 
con pH 4; además de hacer evidente la baja actividad microbicida del ácido clorhídrico. Al emplear un producto comercial que incluía ácido fórmico y propiónico en concentraciones de $50 \mathrm{mM}$ de ácido fórmico, se pudo sensibilizar a las bacterias por un proceso osmótico (Sánchez et al., 2009); sin embargo, se puede deducir que es más importante el nivel de $\mathrm{pH}$, como criterio en la dosificación de los ácidos que la concentración molar de éstos.

Este comportamiento en los pollos del T3, contrasta con el papel primario de los argumentos de modulación microbiana mencionados, lo que sugiere como más importante el efecto que tienen los $\mathrm{AO}$ sobre el proceso digestivo, ya que las aves no fueron desafiadas a ningún patógeno, ni presentaron ningún proceso patológico durante el periodo de prueba. Primeramente, en el proceso digestivo, la acidificación del medio permite una mayor transformación del pepsinógeno en pepsina a nivel del proventrículo y molleja (Cuca et al., 2009) y la digestión proteica es más eficiente; de igual manera el trabajo a nivel del páncreas se ve mejorado mediante el incremento de sus secreciones, y en su caso mejor actividad de algunas enzimas exógenas, como pueden ser las fitasas y mananasas (Rafacz et al., 2005).

Angel et al., (2013) hacen referencia sobre un trabajo donde analizan el efecto del pH del agua sobre el pH del TGl, al comparar dos niveles (8.1 vs. 5.8), donde se tuvo un efecto sobre el ambiente ácido de cada uno de los segmentos del intestino, y como consecuencia el grupo con agua acidificada a $\mathrm{pH}$ 5.8, afecta de manera positiva la digestión de la materia seca; así como la digestibilidad ileal aparente de fósforo. Los autores explican que parte de la menor digestión en el pH alcalino, es por menor efectividad de las fitasas incluidas en la dieta. Cuando el pH aumenta por encima de 4, la eficiencia de éstas, tiende a disminuir por su pH óptimo de actuación y por la precipitación de los quelatos fitato-calcio. Por otro lado, la absorción de calcio es favorecida por la acidificación del medio intestinal (por mantener las sales de calcio en solución), los ácidos carboxílicos como el propiónico y fórmico, reaccionan con los carbonatos para formar sales solubles en agua y ácido carbónico (Brown, 2002), que favorece el crecimiento y fortaleza del sistema óseo (Nourmohammadi, 2013).

En el presente trabajo se observó una mayor resistencia en el rompimiento del yeyuno con una acidificación en el T3 a un pH de 6 . Se han reportado los beneficios que tiene la mejor absorción de minerales; en primer lugar, el calcio para el caso del tejido óseo y de los minerales traza como zinc, cobre y magnesio; los cuales juegan un papel importante en la formación del tejido conectivo. Sin olvidar otras funciones que desempeñan de manera natural los $\mathrm{AO}$ en el metabolismo, como fuente de energía a las células del epitelio intestinal (Boroojeni et al., 2014; Broom 2015; Yang et al., 2019), y sus efectos benéficos en el sistema inmune del tracto gastrointestinal, como la activación de linfocitos y macrófagos; así como un mayor desarrollo del timo y bolsa de Fabricio (Ghulam et al., 2013; Kim et al., 2013; Emami et al., 2017; Al-Mutairi et al., 2020). 
Los resultados del presente estudio con respecto a la histo-morfología del intestino, no muestran una relación clara entre las diferencias de cada tratamiento con respecto a las longitudes evaluadas (largo, ancho y cripta), sin embargo al analizar el área calculada del duodeno, se apreció una menor superficie en el grupo con mejores resultados zootécnicos (T3), al respecto se tiene referencia que el efecto que tienen los $\mathrm{AO}$ en la morfología del intestino delgado ha sido demostrado por varios investigadores, los cuales en su mayoría han encontrado una mayor longitud de las vellosidades en diferentes segmentos del tracto gastrointestinal, con una correlación importante con peso corporal y eficiencia alimenticia (Nourmohammadi, 2013; Emami et al., 2017; Yang et al., 2019), sin embargo, García et al., 2007, reportaron mayor altura de las vellosidades de los grupos adicionados con $\mathrm{AO}$ sin cambios en el área calculada de la vellosidad, pero sí con efecto significativo $(\mathrm{P}<0.01)$ sobre eficiencia alimenticia y digestibilidad. Es importante señalar que una mayor altura de la vellosidad intestinal no es sinónimo de mayor superficie de absorción (Yamauchi, 2007); las variaciones en el ancho suelen estar relacionada con el grado de celularidad, ya que a mayor ancho; mayor infiltración de células del tipo inflamatorio ubicado principalmente en la lámina propia (Maisonnier et al., 2003). De manera que en el presente estudio las aves que fueron tratadas con agua de bebida acidificada con un $\mathrm{pH}$ de 6 , mostraron una menor área calculada de las vellosidades, lo que coincide con los resultados zootécnicos del trabajo de García et al., 2007; lo que sugiere poca celularidad, características de una estructura funcional y sin procesos inflamatorios. La acidificación en el agua de bebida, también afectó los niveles del $\mathrm{pH}$ sanguíneo, entre los tratamientos evaluados $(\mathrm{p}<0.02)$; ya que los valores fueron menores en las aves que estuvieron con $\mathrm{AO}$. Se ha demostrado la influencia que puede tener la alimentación sobre el pH sanguíneo, que aunque existe un mecanismo que promueve el equilibrio a la neutralidad. En el caso de los ácidos en solución permiten el paso de los hidrogeniones a través de las membranas biológicas, este fenómeno se basa en los mecanismos de absorción digestiva y los intercambios iónicos entre los compartimentos digestivos y sanguíneos (Sturkie, 2000; Meschy, 2015). Esta posibilidad de paso de hidrogeniones abre una alternativa en el control de problemas de alcalosis metabólica causada por hiperventilación de las aves en estados de estrés térmico (Sturkie, 2000).

\section{CONCLUSIONES E IMPLICACIONES}

La acidificación del agua de bebida con $0.3 \mathrm{~L} / 1000 \mathrm{~L}$ de $\mathrm{AO}$ (ácido fórmico $31 \%$, ácido propiónico $19 \%$, formato de amonio $26 \%$ y propionato de amonio $6 \%$ ), tuvo un efecto positivo sobre el comportamiento productivo en el pollo de engorda; su efecto benéfico radica en facilitar de manera primaria el proceso de digestión, donde una acidificación moderada a un $\mathrm{pH}$ de 6 es suficiente para lograr un mejor peso corporal y eficiencia alimenticia. La acidificación en el agua de bebida con AO a $0.03 \mathrm{~L} / 1000 \mathrm{~L}$ disminuyó el $\mathrm{pH}$ sanguíneo, por lo que abre una alternativa en el control de problemas de alcalosis 
metabólica, que puede ser causada por hiperventilación de las aves en estados de estrés térmico.

\section{LITERATURA CITADA}

ADHIKARI P, Yadav S, Cosby DE, Cox NA, Jendza JA, Kim WK. 2020. Research Note: Effect of organic acid mixture on growth performance and Salmonella Typhimurium colonization in broiler chickens. Poultry Science. 99(5):2645-2649. https://doi.org/10.1016/j.psj.2019.12.037

ADIL S, Banday T, Bhat GA, Salahuddin M, Raquib M, Shanaz S. 2011 Response of broiler chicken to dietary supplementation of organic acids. Journal of Central European Agriculture. 12(3):498-508. ISSN: 1330-7142. https://doi.org/10.5513/JCEA01/12.3.947

AL-MUTAIRI HMS, Hussein EOS, El Nabi AR, Swelum AA, El-Hack MEA, Taha AE, AlMufarrej SI. 2020. Does the Consumption of Acidified Drinking Water Affect Growth Performance and Lymphoid Organs of Broilers?. Sustainability. 12(8): 3093; https://doi.org/10.3390/su12083093

ANGEL R, Kim SW, Li W, Jimenez-Moreno E. 2013. Velocidad de paso y pH intestinal. Madrid; Memorias del XXIX Curso de Especialización FEDNA. España.

ARAUJO RGAC, Polycarpo GV, Barbieri A, Silva KM, Ventura G, Polycarpo VCC. 2018. Performance and economic viability of broiler chickens fed with probiotic and organic acids in an attempt to replace Growth-Promoting Antibiotics. Brazilian Journal of Poultry Science. 21(2): 1-8. http://dx.doi.org/10.1590/1806-9061-2018-0912

AVILA E. 2018. "Alimentación avícola y del pollos de engorda”. En Ávila GE. Introducción a la zootecnia del pollo y la gallina. CDMX, México: Universidad Nacional Autónoma de México. 468 p. ISBN 978-607-02-9855-4.

BOROOJENI FG, Vahjen W, Mader A, Knorr F, Ruhnke I, Röhe I, Hafeez A, Villodre C, Männer K, Zentek J. 2014. The effects of different thermal treatments and organic acid levels on nutrient digestibility in broilers. Poultry Science. 93: 1440-1452. https://doi.org/10.3382/ps.2013-03563

BOURASSA DV, Wilson KM, Ritz CR, Kiepper BK, Buhr RJ. 2018. Evaluation of the addition of organic acids in the feed and/or water for broilers and the subsequent recovery of Salmonella Typhimurium from litter and ceca1. Poultry Science. 97(1): 64-73. https://doi.org/10.3382/ps/pex289

BROOM LJ. 2015. Organic acids for improving intestinal health of poultry. World's Poultry Science Journal. 71(4), 630-642. https://doi.org/10.1017/S0043933915002391 
BROWN WH.2002. Química Orgánica. 2ª ed. Ciudad de México, México: ed. Patria. Pp. 313-320. ISBN 9789702602088.

CUCA GM, Ávila GE, Pro MA. 2009. "La alimentación aviar es crítica”. En Cuca GM. Alimentación de las aves. Edo. México, México: Universidad Autónoma de Chapingo. Pp.10. ISBN: 978-607-12-0038-9.

DIBNER J, Buttin P. 2002. Use of organic acid as a model to study the impact of gut microflora on nutrition and metabolism. Applied Poultry Research. 11:453-463. ISSN: 1056-6171. https://doi.org/10.1093/japr/11.4.453

EMAMI NK, Daneshmand A, Naeini ZS, Graystone EN, Broom LJ. 2017. Effects of commercial organic acid blends on male broilers challenged with $E$. coli K88: Performance, microbiology, intestinal morphology, and immune response. Poultry Science. 96(9); 3254-3263. https://doi.org/10.3382/ps/pex106

FREITAG M. 2007. Organic acids and salts promote performance and health in animal husbandry. Christian Lücktädt Editor, Acidifiers in Animal Nutrition $1^{\text {st }}$ ed. United Kingdom. Nottingham University Press. Pp.1-7. ISBN-978-189904-3477.

GARCÍA V, Catalá-Gregory P, Hernandez F, Megías M, Madrid J. 2007. Effect of formic acid and plant extracts on growth, nutrient digestibility, intestine mucosa morphology and meat yield broilers. Journal Applied Poultry Research. 16(4): 555-562. ISSN: 1056-6171. https://doi.org/10.3382/japr.2006-00116

GHULAM A, Sohail HK, Habibub-ur R. 2013. Effects of formic acid administration in the drinking water on production performance, egg quality and immune system in the layers during hot season. Avian Biology Research. 6(3):227-232. ISSN: 1758-1567. https://doi.org/10.3184/175815513X13740707043279

IMMERSEEL V, Russell J B, Flythe MD, Gantois I, Timbermont L, Pasmans F, Ducatelle R. 2006. The use of organic acids to combat Salmonella in poultry: a mechanistic explanation of the efficacy. Avian Pathology. 35(3):182-188. ISSN: 0307-9457. https://doi.org/10.1080/03079450600711045

JIN LZ, Ho YW, Abdullah N, Jalaludin S. 1998. Acid and bile tolerance of lactobacillus isolated from chicken intestine. Letter Applied Microbiology. 27(3):183-185. ISSN: 32558254. https://doi.org/10.1046/j.1472-765X.1998.00405.x 
KHAN HS, lqbal J. 2016. Recent advances in the role of organic acids in poultry nutrition. Journal of Applied Animal Research. 44(1):359-369. http://dx.doi.org/10.1080/09712119.2015.1079527

KIM MH, Kang SG, Park JH, Yanagisawa M, Kim CH. 2013. Short-chain fatty acids activate GPR41 and GPR43 on intestinal epithelial cells to promote inflammatory responses in mice. Gastroenterology. 145(2):6-406. ISSN: 0016-5085. https://doi.org/10.1053/j.gastro.2013.04.056

MAISONNIER S, Gomez J, Brée C, Baeza E, Carré B. 2003. Effects of microflora status, dietary bile salts and guar gum on lipid digestibility, intestinal bile salts and histomorphology in broiler chickens. Poultry Science. 82(5):805-814. ISSN: 0032-5791. https://doi.org/10.1093/ps/82.5.805

MEIMANDIOPUR A, Soleimanifarjam A, Azhar A, Hair-Bejo K, Shuhaimi M, Nateghi M, Yazid AM. 2011. Age Effect on short chain fatty acids concentrations and $\mathrm{pH}$ values in the gastrointestinal tract of broiler chicken. Archiv Fur Geflugelkunde. 75(3):164-168. ISSN: 0003-9098. https://www.european-poultry-science.com/Age-effects-on-shortchain-fatty-acids-concentrations-and-pH-values-in-the-gastrointestinal-tract-of-broilerchickens,QUIEPTQyMjAxMzcmTUIEPTE2MTAxNA.html

MESCHY F. Balance Electrolítico y Productividad en Animales. XIV Curso de Especialización: Avances en nutrición y Alimentación animal, FEDNA España. 95-108 p. http://www.fundacionfedna.org/publicaciones_1998

MORTADA M, Cosby DE, Shanmugasundaram R, Selvaraj RK. 2020. In vivo and in vitro assessment of commercial probiotic and organic acid feed additives in broilers challenged with Campylobacter coli. Journal of Applied Poultry Research. 29(2): 435-446. https://doi.org/10.1016/j.japr.2020.02.001

Municipio de Charo. 2020. Medio físico del municipio de Charo, Michoacán. https://www.charo.gob.mx/tu-municipio/medio-fisico. Consultado en 2020.

NAKAI S, Siebert K. 2003. Validation of bacterial growth inhibition models based on molecular properties of organic acids. International Journal Food Microbiology. 86(3):1-7. https://doi.org/10.1016/S0168-1605(02)00551-2 
NGUYEN GH, Lee KY, Mohammadigheisar M, Kim IH. 2018. Evaluation of the blend of organic acids and medium-chain fatty acids in matrix coating as antibiotic growth promoter alternative on growth performance, nutrient digestibility, blood profiles, excreta microflora, and carcass quality in broilers. Poultry Science. 97(12): 4351-4358. https://doi.org/10.3382/ps/pey339

NOM-033-ZOO-1995. 1995. Sacrificio humanitario de los Animales. Domésticos y Silvestres. Diario Oficial de la Federación, Inciso 7.4.2.2. http://www.dof.gob.mx/nota_detalle.php?codigo=5405210\&fecha=26/08/2015

NOURMOHAMMADI R, Afzali N. 2013. Effect of citric acid and microbial phytase on small intestinal morphology. Italian Journal Animal Science. 12(1):44-47. ISSN: 1828-051X. https://doi.org/10.4081/ijas.2013.e7

POLYCARPO GV, Andretta I, Kipper M, Cruz-Polycarpo VC, Dadalt JC, Rodrigues PHM, Albuqueque R. 2017. Meta-analytic study of organic acids as an alternative performanceenhancing feed additive to antibiotics for broiler chickens. Poultry Science. 96(10): 36453653. https://doi.org/10.3382/ps/pex178

RAFACZ-LIVINGTON KA, Parsons CM, Jungkt RA. 2005. The effects of various organic acids on phytate phosphorus utilization in chicks. Poultry Science. 84(9):1356-1362. ISSN: 0032-5791. https://doi.org/10.1093/ps/84.9.1356

RICKE S. 2003. Perspectives on the use of organic acids and short chain fatty acids as antimicrobials. Poultry Science. 82:632-639. ISNN:0032-5791, https://doi.org/10.1093/ps/82.4.632

ROTH N, Hofacre C, Zitz U, Mathis GF, Molder K, Doupovec B, Berghouse, Doming KJ. 2019. Prevalence of antibiotic-resistant $E$. coli in broilers challenged with a multi-resistant E. coli strain and received ampicillin, an organic acid-based feed additive or a synbiotic preparation. Poultry Science. 98(6): 2598-2607. https://doi.org/10.3382/ps/pez004

ROTH N, Mayrhofer S, Gierus M, Weingut C, Schwarz, Doupovec B, Berrios R, Domig JK. 2017. Effect of an organic acids-based feed additive and enrofloxacin on the prevalence of antibiotic-resistant $E$. coli in cecum of broilers. Poultry Science. 96(11):4053-4060. https://doi.org/10.3382/ps/pex232 
SÁNCHEZ-HERRERA I, Posadas HE, Sánchez RE, Fuente MB, Hernández EJ, Laparra VJL, Avila GE. 2009. Efecto del butirato de sodio en dietas para gallinas sobre el comportamiento productivo, calidad del huevo y vellosidades intestinales. Veterinaria México. 40(4):397-403. ISSN: 0301-5092.

http://www.scielo.org.mx/scielo.php?script=sci_arttext\&pid=S0301-50922009000400006

SAS Institute. 2010. Statistical Analyses Software. SAS/STAT ${ }^{\circledR}$ version 9.0.2, Cary, N.C., USA: SAS Institute Inc., ISBN: 978-1-60764-599-3. http://www.sas.com/en_us/software/analytics/stat.html\#

SMITH JA. 2011. Experiences with drug-free broiler production. Poultry Science. 90:26702678. ISSN:0032-5791. https://doi.org/10.3382/ps.2010-01032

WHITTOW G. 2000. Sturkie's Avian Physiology. 5th ed. San Diego, California: Ed. Academic Press. Pp. 298. ISBN: 9780127476056

YAMAUCHI K. 2007. Review of a histological intestinal approach to assesing the intestinal function in chickens and pigs. Animal Science Journal. 78(4):356-370. ISSN: 1525-3163. https://doi.org/10.1111/j.1740-0929.2007.00448.x

YANG X, Liu Y, Yan F, Yang C, Yang X. 2019. Effects of encapsulated organic acids and essential oils on intestinal barrier, microbial count, and bacterial metabolites in broiler chickens. Poultry Science. 98(7): 2858-2865. https://doi.org/10.3382/ps/pez031 